\title{
Domains of fatigue are associated with poor performance and sarcopenia in older Scottish adults
}

\author{
L. Wyness, F. Lemmon, I. Arvanitidou, E. Bannerman, I. Davidson and J. Jones \\ Queen Margaret University, Edinburgh EH21 6UU, UK
}

There has been a growing interest in fatigue as an early marker of age-related decline in health and functional abilities ${ }^{(1)}$. Fatigue has been associated with poor functional performance and has been shown to be predictive of functional decline in older adults ${ }^{(2)}$, however there is little published research linking fatigue to sarcopenia and results are inconsistent ${ }^{(3)}$. The aim of this study was to determine the association of fatigue on performance and incidence of sarcopenia among older adults in Scotland.

The study included 59 community dwelling adults aged 65+ years. Data was collected on: age and body mass index (BMI) Domains of self-reported fatigue were measured using the Multidimensional Fatigue Inventory (MFI) ${ }^{(4)}$. The questionnaire consists of 20 statements relating to five aspects of fatigue: general, physical and mental fatigue and degree of reduced activity or motivation. Physical performance was assessed by gait speed (measured over a 4-meter course) and muscle strength was assessed by handgrip strength.

The older adults $(80+$ years $n=40)$ in this sample had a significantly slower gait speed than the young older adults $(65-79$ years $\mathrm{n}=19)$. BMI and hand grip strength were both significantly lower among the older adults.

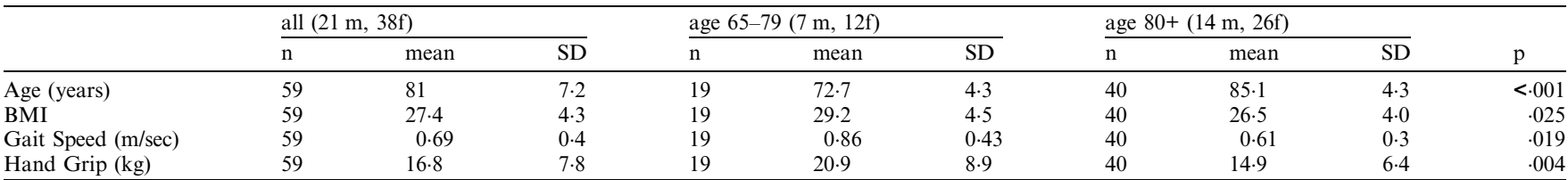

Independ

Logistic regression revealed a slower gait speed was associated with higher scores for physical fatigue $(p=0.022)$, reduced activity $(p=0.006)$ and reduced motivation $(p=0.004)$. The presence of sarcopenia was associated with reduced motivation $(p=0.015)$. Assessment/screening of domains of fatigue may provide a useful index for the presence of sarcopenia in older adults.

1. Avlund K (2010) Aging Clin Exp Res 22, 100-15.

2. Avlund K, Vass M, Hendriksen C (2003) Age Ageing 32, 579-84.

3. Cooper C, Fielding R, Visser M et al. (2013) Calcif Tissue Int 93, 201-10.

4. Smets EMA, Garssen B, Bonke B and Haes de JCJM. (1995) J Psychosom Res 39, 315-25. 